\title{
Learning imaginary rotation
}

\author{
LLOYD R. PETERSON and TIMOTHY FEUSTAL \\ Indiana University, Bloomington, Indiana
}

\begin{abstract}
Imaginary rotation of patterns of five filled cells in a $3 \times 3$ grid was investigated. Accuracy was found to drop off markedly with degree of rotation, but it improved with practice. Symmetry of pattern was a significant variable. Transfer of training to different patterns was substantial, and it was concluded that subjects were learning to rotate, as distinct from memorizing patterns. Imagining was discussed as a skill.
\end{abstract}

The role of learning in mental rotation has received relatively little attention. Indeed, some experimenters have minimized the importance of learning, as witness the comment that "it is doubtful that practice has any substantial effect on the rate of mental rotation" (Cooper \& Podgorny, 1976, p. 510). Cooper and Podgorny followed traditional procedures of reporting performances only after a steady state was presumed to have been reached.

Consider the view that imagery is a skill (Kolers \& Smythe, 1979). In terms of the classical analysis of skill learning into three stages, most mental-rotation studies seem to have concerned themselves with the third stage of imagining, one characterized by an absence of errors and rapid automatic performance. The present paper attempts an analysis of early practice on mental rotation.

Pylyshyn (1979) presented evidence that reaction time in a mental-rotation task speeds up during several days of practice. Of course, because such a task involves more than rotation, decreasing reaction times do not necessarily indicate a change in the rate of rotation per se. What is learned in recognition tests may be a set of transformed patterns for each standard pattern. On the other hand, improvement could result from learning to rotate, a generalized learning not confined to specific patterns. The measurement of this type of learning was the objective of the current experiment, and patternspecific learning was viewed as a factor to be minimized. A substitute for the recognition technique was sought. Since recognition testing requires the display of patterns in their transformed orientations, such displays might be expected to facilitate their memorization. Therefore, subjects were told to type a description of a pattern which had never been seen save in the imagination, rather than being shown patterns to recognize.

A test began by display of a pattern in standard orientation. After the display ended a cue was given to rotate the pattern to some new orientation. The subject was then asked to describe it without ever seeing it in

Lloyd R. Peterson is with the Department of Psychology, Indiana University, Bloomington, Indiana 47405. Timothy Feustal is now with American Bell, Lincroft, New Jersey 07738. this transformed state. In order to facilitate description, patterns were based on a 3 by 3 grid which could be described cell by cell by pressing one of two keys to indicate a filled or empty cell (Peterson, Rawlings, \& Cohen, 1977). After practice with one set of patterns, transfer to a new set was tested. Learning to imagine, as distinct from pattern-specific learning, was inferred from performance on this transfer test.

\section{METHOD}

Fifteen subjects from introductory psychology classes were run individually. Three of these were dropped in initial practice tests due to their failure to perform adequately. The subjects were seated before a Teletype; their eyes were approximately $90 \mathrm{~cm}$ from a panel displaying a $3 \times 3$ array of white light bulbs. By illuminating subsets of the array, patterns were presented. The panel also held red lights. The top red light signaled a 0 -deg rotation, the red light on the right indicated a 90-deg rotation, and the bottom light a $180-\mathrm{deg}$ rotation.

The display and teletype were controlled by a PDP-8/L digital computer. The subject responded by pressing one of two keys, one labeled ON (for a lighted cell) or one labeled OFF (for a dark cell). The computer accepted keypresses only after the red light had flashed on. The pattern was described in the following order: the upper left corner, across the top row, followed by the middle and bottom rows. The subjects could restart their descriptions by pressing a yellow key.

The patterns were chosen from those that Handel and Garner (1966) had scaled for goodness. Two sets were constructed such that mean goodness was equated. Within a set, there were three patterns symmetrical on one axis and three asymmetrical patterns.

\section{Procedure}

Initial instructions led to practice in describing patterns based on other than five lights. The bell of the Teletype preceded a $3-\mathrm{sec}$ pattern display by $.01 \mathrm{sec}$. Six practice tests were given, and if more than two errors occurred, the set of tests was repeated.

In Block 1 , the subjects received 14 nonrotation tests on 14 Garner figures, including those to be shown later plus two others. The subjects were instructed to concentrate on imagining the pattern while it was displayed, and continuing to imagine it during a 10 -sec unfilled interval that followed. When the red light on top of the display board flashed, description of the pattern by keypresses was to begin.

In Block 2, rotation training was instituted for 18 tests. Instructions included demonstration of a three-light pattern shown in $0-, 90-$, and $180-\mathrm{deg}$ orientations. In the tests that 
followed, three symmetrical and three asymmetrical patterns were each presented in the three orientations. Order of the patterns and orientations was randomized for individual subjects. Rotation time was measured from onset of the red light signaling the required orientation to the subject's first keypress. The description was scored correct if the last nine keypresses before the terminator (space bar) were all in the proper order.

Block 3 followed after an interval of 2 or 3 days. Rotation practice (18 tests on the same patterns in another random order) similar to the previous block was given.

Block 4 constituted a transfer test. The subject was told that different patterns were to be rotated, patterns that had been seen before near the beginning of the experiment. A subject now received a set of patterns different from the patterns presented in Blocks 2 and 3. The order of sets was counterbalanced over the group of subjects.

\section{RESULTS}

The accuracy of descriptions in the nonrotation recall of Block 1 was quite good, as can be seen in Figure 1. However, when rotation practice began in Block 2, performance deteriorated markedly. Note that the symmetrical patterns were rotated successfully more often than were the asymmetrical patterns. Continued practice with these same patterns through Block 3 resulted in some improvement in performance from Block 2 to Block 3. Finally, in the transfer test of Block 4, there was further improvement over Block 3 in three of four conditions.

\section{ASYMMETRIC PATTERNS}

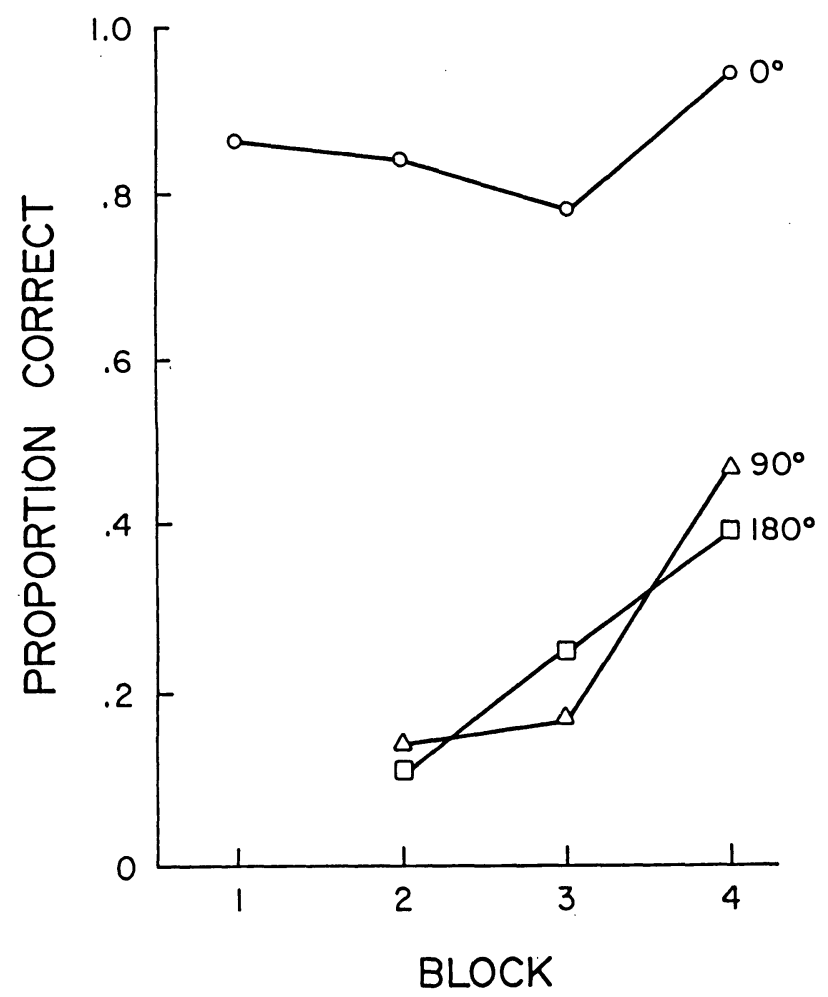

An analysis of variance over Blocks $2-4$ corroborated the above summary. Significant at the .01 level were block $[\mathrm{F}(2,22)=11.76]$, symmetry $[\mathrm{F}(1,11)=89.22]$, and degree $[F(2,22)=32.86]$. The interaction of symmetry and degree was also significant $[\mathrm{F}(2,22)=27.25]$. An analysis including only Blocks 2 and 4 was significant $[F(1,11)=18.89]$. Performance on the transfer patterns was significantly better than initial rotation performance on the original patterns. The analysis of variance on only the data from Blocks 3 and 4 was also significant for block $[F(1,11)=18.9]$.

\section{DISCUSSION}

There were two findings that had not been anticipated in the experiment. First, the magnitude of the drop in performance at the onset of rotation practice was larger than had been anticipated. There seemed to be no difficulty in holding the static image of the pattern over a 10-sec interval in Block 1 , even though the subjects had never seen the patterns before. Perhaps this retention interval could be better described as a rehearsal interval. In any event, the performance on rotation tests is clearly not due to the passage of time per se. The subjects experienced difficulty in rotation, not in holding and describing the patterns per se. Symmetry of the pattern was a robust factor in difficulty of performing the rotational transformation. Indeed, this was the only evidence for pattern-specific effects in the data.

The second unexpected finding was the amount of transfer with the introduction of new patterns. We looked for improve-

\section{SYMMETRIC PATTERNS}

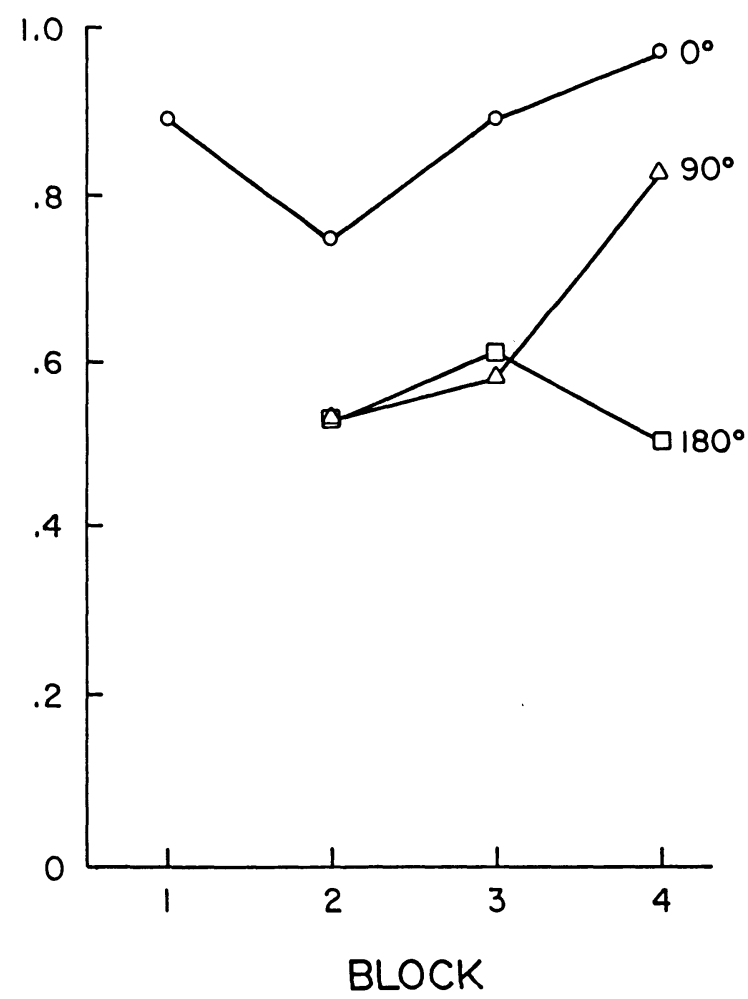

Figure 1. Proportions of correct descriptions of asymmetric and symmetric patterns after varying degrees of imaginary rotation. 
ment during Block 4 over Block 2, but assumed there would be some decrement from Block 3 to Block 4. The finding that there was improvement even in the comparison of Block 4 with Block 3 for three of four conditions was not anticipated. Clearly, there was a strong general transfer effect. Of course, learning to learn can arise through a variety of factors, including a subject's becoming accustomed to a difficult response routine. In the present case, the general aspects of the experimental situation did not seem difficult until the rotation requirement was introduced. It seems likely that general learning of procedures was acquired quickly in the opening stages of the experiment. The succeeding learning to learn would then have been related to the rotation per se.

The data are consistent with the interpretation that, when rotation was first required, these subjects engaged in relatively slow tiral-and-error efforts similar to those that characterize the initial stage of skill learning. The majority had to acquire facility in the task by laborious effort. Some of these subjects could be heard over the intercom talking to themselves about the difficulty of the task. Smooth rotation at a constant rate was not typical. Such performance would have to wait on a more advanced stage of practice, beyond that achieved in the present study.

\section{REFERENCES}

Cooper, L. A., \& Podgorny, P. (1976). Mental transformations and visual comparison processes: Effects of complexity and similarity. Journal of Experimental Psychology: Human Perception and Performance, 2, 503-514.

HANDel, S., \& Garner, W. R. (1966). The structure of visual pattern associates and pattern goodness. Perception \& Psychophysics, 1, 33-38.

Kolers, P. A., \& Smythe, W. E. (1979). Images, symbols, and skills. Canadian Journal of Psychology, 33, 158-184.

Peterson, L. R., Rawlings, L., \& Cohen, C. (1977). The internal construction of spatial patterns. In G. Bower (Ed.), The psychology of learning and motivation (Vol. 11). New York: Academic Press.

Pylyshyn, Z. W. (1979). The rate of "mental rotation" of images: A test of the holistic analogue hypothesis. Memory \& Cognition, 7, 19-28.

(Manuscript received for publication October 31, 1983.) 